\title{
Control of Center Wavelength in Reflective-Arrayed Waveguide-Grating Multiplexers
}

\author{
L. Grave de Peralta, Ayrton A. Bernussi, V. Gorbounov, J. M. Berg, and H. Temkin, Fellow, IEEE
}

\begin{abstract}
A new approach to compensate for the channel-wavelength shift due to fabrication errors and thermal effects in arrayed waveguide-grating multiplexers is described. The method combines a silica-based reflective multiplexer with a composite mirror made of materials with different coefficients of thermal expansion. Differential thermal expansion of the mirror assembly rotates its reflecting surface at a constant rate with temperature, compensating for the temperature-induced changes in the effective index of refraction of the waveguide material. The use of external mirror also allows for wavelength trimming that centers the channel wavelength at the standard grid. The channel wavelength can be tuned by up to $2 \mathrm{~nm}$ without increased insertion loss or changes in channel-to-channel separation. The channel wavelength shifts linearly with the external mirror angle at a rate of $\sim 66 \mathrm{~nm} / \mathrm{deg}$ in excellent agreement with simulation. A finite element analysis of the composite mirror shows negligible deformation of the reflecting surface over a wide range of temperatures, in good agreement with experimental results.
\end{abstract}

Index Terms-Arrayed waveguide grating (AWG), athermal operation, multiplexer, silica waveguides, wavelength division multiplexing.

\section{INTRODUCTION}

A CCURACY of the center channel wavelength relative to the standard ITU grid is one of the key performance parameters of arrayed waveguide-grating (AWG) devices [1]. Wavelength deviations arise from the accumulation of errors and inaccuracies in the fabrication process and the problem is particularly acute in devices with very narrow passband response, such as Gaussian AWGs. Moreover, in silica-based AWGs, the channel peak wavelength shifts as a result of the temperature dependence of the refractive index of the waveguide [2]. In conventional AWGs, deviations of the channel wavelength related to fabrication errors can be corrected by selecting the appropriate input waveguide, a procedure known as the vernier method [2], but this cannot be applied to reflective AWGs (R-AWGs) with a single input channel [3], [4]. A common approach to compensate for the temperature-induced channel wavelength shift is to use a thermoelectric element to temperature-tune the device response. This requires provision of electric power to the device, accurate temperature control,

Manuscript received April 8, 2004; revised July 30, 2004. This work was performed at Texas Tech University, Lubbock, and was supported in part by Texas' Technology Development and Transfer program and the Jack F Maddox Foundation. The work of L. Grave de Peralta and A. A. Bernussi was supported in part by NSF-SBIR under Grant 0339012.

L. Grave de Peralta and A. A. Bernussi are with the Multipass Corporation, Ransom Canyon, TX 79366 USA, and also with the Nano Tech Center, Texas Tech University, Lubbock, TX 79409 USA (e-mail: luisgrave@ hotmail.com).

V. Gorbounov, J. M. Berg, and H. Temkin are with the Nano Tech Center, Texas Tech University, Lubbock, TX 79409 USA.

Digital Object Identifier 10.1109/JQE.2004.837789 and results in a more complex package. Alternative solutions to prepare temperature-insensitive AWGs thus continue to be proposed [5]-[7] but all of them require fairly complex modifications in the device design or to the fabrication process.

In R-AWGs, the channel wavelength depends on the angle between the waveguides and the reflecting surface terminating the grating [8]. A change in this angle alters the path-length difference between consecutive waveguides of the grating. However, this angular sensitivity, an apparent disadvantage of the R-AWG design, can be used to correct the center channel wavelength and, at the same time, make the device insensitive to temperature variations. We show that this can be accomplished by combining an R-AWG with an external mirror, placed closely, and at a precise angle, with respect to the surface terminating the grating. By adjusting the angle of the external mirror, the channel wavelength can be tuned to match the ITU grid. The composite external mirror is formed with materials of different thermal expansion coefficients, resulting in a constant rate rotation of its reflecting surface with temperature [8]. This rotation compensates the temperature-induced changes in the effective index of refraction of the waveguide, resulting in athermal operation.

We present detailed experiments and simulations demonstrating the use of an external mirror to control the channel wavelength and suppress the temperature sensitivity of silica-based R-AWGs. Our results show that the channel wavelength can be tuned by up to $2 \mathrm{~nm}$ without significant changes in the device performance. The observed linear shift of the channel wavelength and the negligible change in the output channel separation of R-AWGs with the mirror angle are in quantitative agreement with simulation results.

Athermal operation of R-AWGs was successfully demonstrated using two designs of the composite mirror and their construction and performance are described. The mirrors are shown to rotate with temperature at a constant rate, in agreement with simulation results. The use of external mirrors reduced the wavelength-temperature sensitivity of these devices from $1.06 \times 10^{-2} \mathrm{~nm} /{ }^{\circ} \mathrm{C}$ to less than $3.0 \times 10^{-4} \mathrm{~nm} /{ }^{\circ} \mathrm{C}$, in the temperature range of $5^{\circ} \mathrm{C}-70^{\circ} \mathrm{C}$. This was accomplished without any degradation in the device performance or any need for modifications in the R-AWG design or in the chip fabrication process.

\section{EXPERIMENTAL DETAILS AND RESUlTS}

\section{A. Silica-Based R-AWGs}

Detailed description of the design, fabrication, and performance of R-AWGs used in this work has been provided previously [9]. Current experiments relied on 40-channel devices with the channel-to-channel spacing of 100-GHz and Gaussian 
(a)

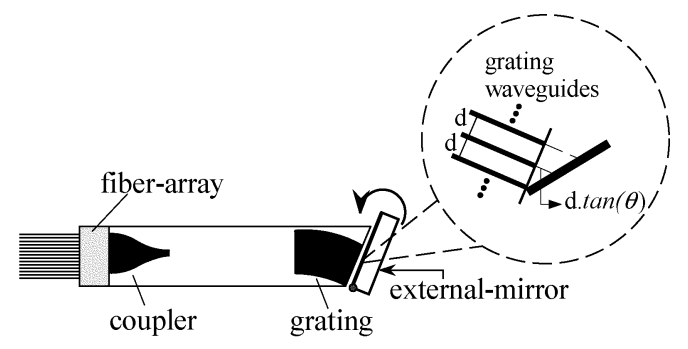

(b)

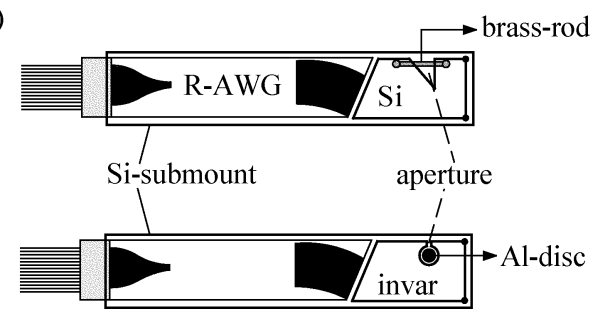

Fig. 1. (a) Configuration used to control the channel wavelength in R-AWGs using a simple external mirror. Inset shows the change in the path length difference between consecutive grating waveguides in the presence of an external mirror (the angle between the waveguides terminating the grating and the external mirror was greatly exaggerated for easier visualization). R-AWGs athermal assembly configurations using composite mirrors. (b) Si-miror with a metal rod attached across an aperture. (c) Invar mirror with an Al plug inserted into a circular aperture.

transmission responses. R-AWGs were fabricated using doped and undoped $\mathrm{SiO}_{2}$ layers deposited on a silicon substrate. The relative refractive index $(n)$ difference between the core and the cladding materials $\left(n_{\text {core }}=1.456\right.$ and $n_{\text {cladding }}=1.446$, at $\lambda=1.55 \mu \mathrm{m}$ ) was $0.68 \%$. In standard operation, high reflectivity of the reflecting surface was assured by the deposition of a Cr-Au film. The R-AWG chip used in our experiments was $9 \mathrm{~cm}$ long and $2 \mathrm{~cm}$ wide. In the external mirror experiments discussed here the $\mathrm{Cr}$-Au layer was removed by diamond polishing.

\section{B. Control of Center Wavelength}

A schematic representation of the experimental approach used to compensate the wavelength shift resulting from fabrication inaccuracies is illustrated in Fig. 1(a). The external mirror (Au film deposited on a glass substrate) was positioned a few microns away from the grating of the R-AWG. The angular tilt of the external mirror was adjusted by placing it on a rotational stage. Index matching material was applied between the external mirror and the AWG in order to minimize losses and to suppress reflections from the waveguide grating-air interface. In the experiment illustrated in Fig. 1(a), rotation of the external mirror results in changes in the path length difference $(\Delta L)$ between consecutive waveguides of the grating, as shown schematically in the inset of Fig. 1(a). In R-AWGs studied in this work, the waveguides of the grating were designed to be perpendicular to the surface terminating the grating. The grating was designed with a constant distance $d \sim 31 \mu$ m between consecutive waveguides at the reflecting surface. An external mirror tilt by an angle $\theta$ results in an additional constant path length difference contribution of $d \tan (\theta)$ to $\Delta L$.

Transmission spectra for output channels \#19 and \#40 of an R-AWG are shown in Fig. 2 for different angular positions of the external mirror. As the angle between the device and the

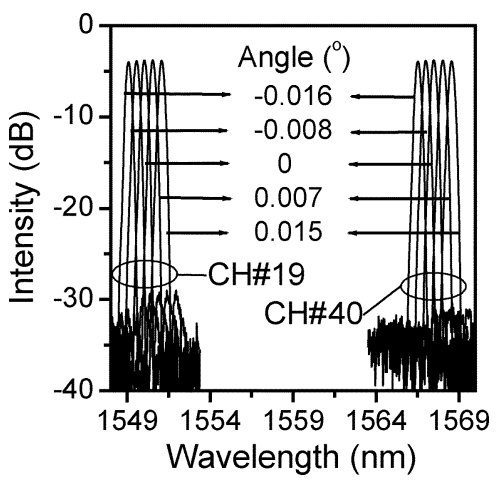

Fig. 2. Transmission spectra of two output channels of an R-AWG at different angles of the external mirror.

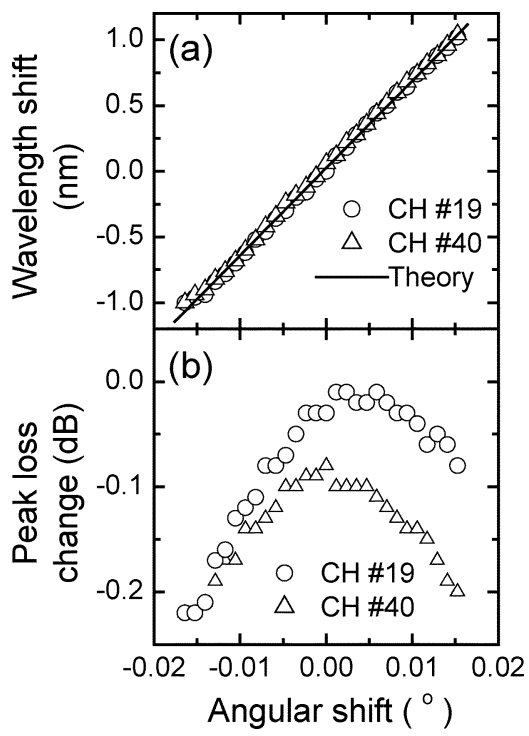

Fig. 3. (a) Channel wavelength shift. (b) Corresponding peak loss change as a function of the angular position of the external mirror for two output channels (CH\#19 and CH\#40) of a single R-AWG. The solid line in part (a) represents the calculated shift of the peak wavelength with the angle of the external mirror.

external mirror is increased, the spectra shift systematically to higher wavelengths. Varying the mirror angle by a total of $\sim 0.03^{\circ}$ results in a wavelength shift of $2.0 \mathrm{~nm}$, for both channels. Similar results were found for other output channels of the device. The corresponding peak wavelength shift and loss change with the angle, for both output channels, are shown in Fig. 3(a) and (b), respectively. The data of Fig. 3(a) shows that the channel peak wavelength changes linearly with the angle of the external mirror. The same slope of $\sim 66 \mathrm{~nm} / \mathrm{deg}$ was determined from both channels. Increased diffraction losses were introduced when the external mirror was tilted with respect to the surface terminating the grating. These losses result from changes in the gap distance between the external mirror and the grating as the tilt angle is varied. This limits the practical center wavelength correction that can be accomplished with an external mirror. A maximum loss change of $\sim 0.22 \mathrm{~dB}$ was determined when the mirror angle was tilted by $\sim 0.03^{\circ}$, as shown in Fig. 3(b). A loss penalty of less than $0.15 \mathrm{~dB}$ was observed for the angular tilt corresponding to the wavelength shift equal to the $0.8-\mathrm{nm}$ channel-to-channel separation. The data shown in Figs. 2 and 3 demonstrate that an external mirror can 


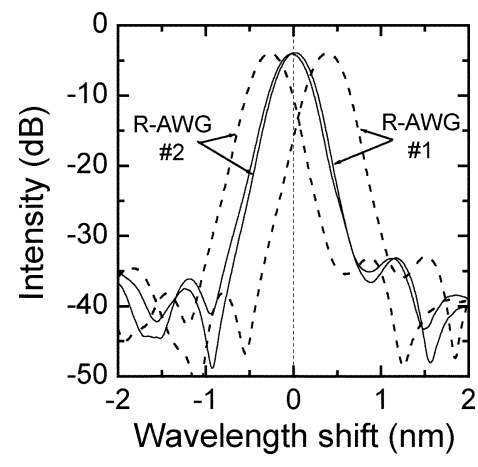

Fig. 4. Transmission spectra of a single output channel, relative to the corresponding ITU channel, for two different R-AWG devices (labeled \#1 and \#2) before (dashed line) and after (solid line) channel wavelength compensation.

be used to compensate for deviations of the channel wavelength due to fabrication errors and inaccuracies, without significant degradation in the device performance.

In order to demonstrate the accuracy of our channel positioning approach we experimented with two R-AWGs exhibiting distinct wavelength deviations from the standard ITU grid. The device and the external mirror were placed on a common Si submount. The R-AWG was attached first with epoxy. The angular position of the external mirror was then adjusted to place the channel wavelength of each device at the ITU grid. Finally, the mirror was attached to the submount. Examples of single channel transmission spectra for each device, before and after wavelength compensation, are shown in Fig. 4. The initial center channel deviations of -0.27 and $+0.39 \mathrm{~nm}$ were reduced to +0.017 and $-0.019 \mathrm{~nm}$, respectively, without any appreciable degradation in the device performance. The peak wavelength precision after wavelength compensation is less than $\pm 0.05 \mathrm{~nm}$, which is the standard requirement for this type of devices [2]. Similar results were found for other channels of both devices.

\section{Temperature-Induced Wavelength Shift Compensation: Composite Mirror Structures}

The previous section described the use of an external mirror to compensate for wavelength shifts in R-AWGs arising from fabrication inaccuracies. However, this does not eliminate wavelength shifts due to temperature-induced changes in the index of refraction of silica waveguides. We showed recently that the thermal sensitivity of silica-based R-AWGs can be suppressed using an external mirror that rotates at a constant rate with the temperature [8]. To accomplish this we designed a composite mirror based on materials with different thermal expansion coefficients. Two different approaches to the fabrication of such composite mirrors, schematically shown in Fig. 1(b) and (c), are described.

The first relies on a composite mirror where a brass-rod (with a cross section area of $\sim 2 \mathrm{~mm}^{2}$ ) was attached with epoxy across a triangular aperture $(\sim 5 \mathrm{~mm}$ wide at the top) machined in $800-\mu \mathrm{m}$-thick Si wafer, as shown in Fig. 1(b) [8]. The mirror was approximately $20 \mathrm{~mm}$ long and $16 \mathrm{~mm}$ wide. The vertex of the v-shaped aperture was placed $8 \mathrm{~mm}$ below the edge, at the center of the body of the mirror, and $10 \mathrm{~mm}$ away from the
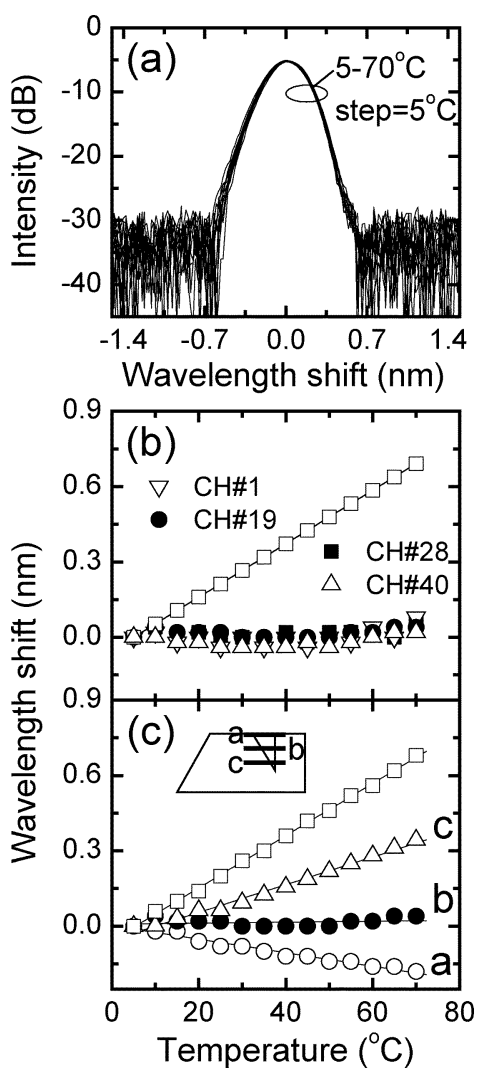

Fig. 5. (a) Transmission spectra as a function of temperature of a single output channel (\#19) of a R-AWG combined with a composite mirror shown in Fig. 1(b), with the metal rod attached to the center of the V-aperture. (b) Peak wavelength shift as a function of temperature for four output channels (\#1, \#19, \#28, and \#40) of an R-AWG. (c) Peak wavelength shift of channel \#19 as a function of temperature for different positions of the metal rod across the aperture. The inset illustrates positions of the metal rod across the aperture. The temperature-induced wavelength shift of the R-AWG with an ordinary mirror is shown ( $\square$ ) for comparison in parts (b) and (c).

reflecting surface. Diamond polishing followed by gold deposition assured high reflectivity of the edge of the $\mathrm{Si}$ wafer, its reflecting surface. The linear expansion coefficient of metals is considerably larger than that of silicon. Consequently, when the temperature is increased (decreased) an elongation (contraction) of the metal rod takes place, tilting the mirror. The device assembly was started by attaching the R-AWG chip to a Si-submount. The external mirror was then positioned $\sim 5 \mu \mathrm{m}$ away from the reflecting surface terminating the grating, at an angular position adjusted to produce the center wavelength matching the ITU grid. The back part of the mirror chip, away from the reflecting surface, was attached to the $\mathrm{Si}$ submount supporting the R-AWG, while the reflecting part was free to rotate.

Transmission spectra of a single output channel (\#19) of an R-AWG using the arrangement illustrated in Fig. 1(b) are displayed in Fig. 5(a) as a function of temperature. In these experiments index-matching material was applied between the composite mirror and the R-AWG and the metal rod was positioned close to the center of the aperture of the mirror. As shown in Fig. 5(a), the spectra are essentially insensitive to changes in temperature. Similar results were obtained for other output channels of the same device. Representative peak wavelength shift for the output channel shown in Fig. 5(a) and for other three different channels are plotted as a function of temperature 
in Fig. 5(b). This demonstrates that efficient athermal operation is obtained for all the output channels. In order to show the effect of the bar position across the mirror aperture in the response of the device, the peak wavelength shift is plotted as a function of temperature in Fig. 5(c). Wavelength shift of an R-AWG using an ordinary (nonrotating) external mirror is included for comparison. When the metal rod was placed at the center of the aperture, a slope as low as $2.3 \times 10^{-4} \mathrm{~nm} /{ }^{\circ} \mathrm{C}$ was measured. This is almost two orders of magnitude lower than the slope of $1.06 \times 10^{-2} \mathrm{~nm} /{ }^{\circ} \mathrm{C}$ exhibited by conventional silica-based AWGs. In the temperature range $5{ }^{\circ} \mathrm{C}-70^{\circ} \mathrm{C}$ a maximum peak wavelength deviation of $0.015 \mathrm{~nm}$ was observed. This value is well below the $\pm 0.05 \mathrm{~nm}$ required for this type of devices [2]. Placing the metal rod at the top (bottom) of the aperture results in over (under) temperature compensation of the transmission response. Peak loss change of less than $0.2 \mathrm{~dB}$ was observed in the temperature range of $5^{\circ} \mathrm{C}-70^{\circ} \mathrm{C}$. The results shown in Fig. 5 demonstrate a temperature insensitive R-AWGs prepared using a composite external mirror that rotates at a constant rate with temperature. This was achieved without any appreciable degradation in the device performance.

A second composite mirror design is illustrated in Fig. 1(c). Rotation of a reflecting surface is again assured by fabricating the mirror from materials with different coefficients of thermal expansion. The mirror body was fabricated from invar alloy with a thermal expansion coefficient similar to that of silicon. Dimensions of the metallic mirror are similar to those of the silicon mirror described above. A circular aperture machined in the body of the mirror was centered at $2.5 \mathrm{~mm}$ below the edge and $10 \mathrm{~mm}$ away from the reflecting surface. An aluminum plug, $3.3 \mathrm{~mm}$ in diameter, serving as the expansion member, was press-fitted into a circular aperture machined in the body of the mirror, removing the need for adhesives. A slot connected the aperture to the edge of the mirror, allowing for rotation of the reflecting surface with temperature.

Output spectra of a single channel (\#19) measured at different temperatures, using the configuration illustrated in Fig. 1(c), produced results similar to those obtained in Fig. 5(a), as shown in Fig. 6(a). Fig. 6(b) shows representative data for the peak wavelength shift of four R-AWG output channels at different temperatures, when the composite mirror illustrated in Fig. 1(c) is used as the external reflector. We include, for comparison, the wavelength shift of the R-AWG using an ordinary external mirror. Similar to the results shown in Fig. 5, athermal operation for all measured output channels was obtained using the metallic mirror. When the temperature was varied in the range of $5^{\circ} \mathrm{C}-70^{\circ} \mathrm{C}$, wavelength change with the slope as low as $-1.6 \times 10^{-4} \mathrm{~nm} /{ }^{\circ} \mathrm{C}$ was determined from the data of Fig. 6(b). A maximum peak wavelength deviation of $0.01 \mathrm{~nm}$ was observed in this temperature range. Fine tuning of the slope of the peak wavelength temperature dependence can be also accomplished with this mirror design. This could be done by changing the material of the plug, by adjusting the plug thickness, or by using ring-shaped plugs with different inner hole diameters.

\section{Theory AND Finite Element SimUlation}

In order to carry out quantitative analysis of the results shown in Figs. 2-6, we modeled the center wavelength of the R-AWG
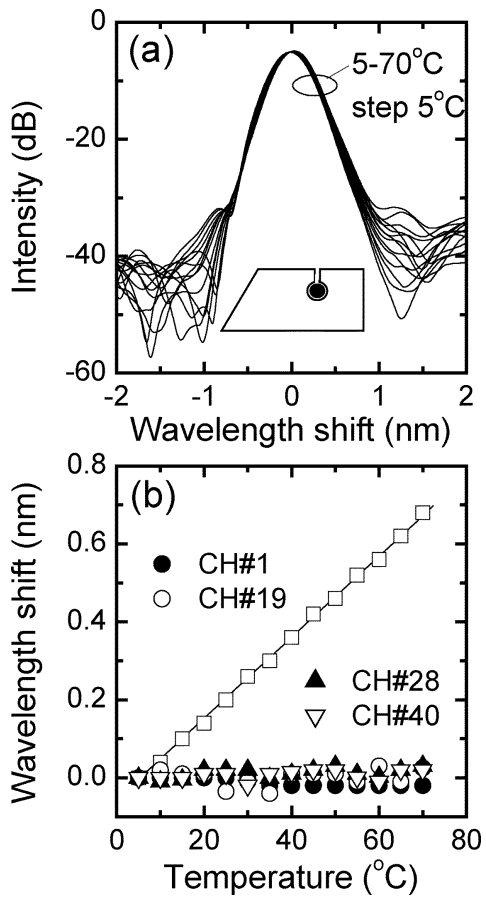

Fig. 6. (a) Transmission spectra as a function of temperature of a single output channel (\#19) of a R-AWG combined with a composite mirror shown in Fig. 1(c). (b) Peak wavelength shift as a function of temperature for four output channels (\#1, \#19, \#28 and \#40) of an R-AWG. The temperature-induced wavelength shift of the R-AWG with an ordinary mirror ( $\square$ ) is shown for comparison.

with an external mirror used as a reflector. The temperatureinduced rotation rates of the reflecting surface, for both types of composite mirrors, were evaluated with a finite element (FE) model that takes into account the materials and the geometry of each mirror.

The center channel wavelength $\left(\lambda_{c}\right)$ of an R-AWG can be described as [2]

$$
\lambda_{c}=2 \frac{n_{c} \Delta L+n_{g} \Delta L_{g}}{m}
$$

where $n_{c} \Delta L$ is the intended optical path-length difference and $n_{g} \Delta L_{g}$ is the additional contribution due to light propagating through the medium filling the gap between the grating and the external mirror [see Fig. 1(a)]. In our case, the optical path length of the medium filling the gap is included by substituting $n_{g} \Delta L_{g} \rightarrow n_{g}\left[\Delta L_{g}+d \tan (\theta)\right]$. The peak wavelengths calculated as a function of the mirror angle for both output channels are shown as a solid line in Fig. 3(a). Excellent agreement between the calculated and measured center peak wavelength dependence on the mirror angle is evident.

The wavelength separation $(\Delta \lambda)$ between consecutive channels also depends on the optical path length difference [2]

$$
\Delta \lambda=\frac{n_{s} d_{c} d_{g} \lambda_{o}}{2 f\left(N_{c} \Delta L+N_{g} \Delta L_{g}\right)}
$$

where $\lambda_{o}$ is the design channel wavelength, $n_{s}$ is the effective refractive index of the slab region, $d_{c}$ is the distance between waveguides at the coupler-slab interface, $d_{g}$ is the distance between waveguides at the grating-slab interface, $f$ is the radius of the slab, and $N_{c}$ and $N_{g}$ are the group refractive index of 
TABLE I

SiLICON MirRor ASSEMBLY SIMULATION PARAMETERS

\begin{tabular}{c|c|c|c|c|c|c|c}
\hline \multicolumn{2}{l|}{ Thickness $(\mathrm{mm})$} & \multicolumn{2}{c|}{ Young's Modulus $(\mathrm{GPa})$} & \multicolumn{2}{c|}{ Poisson's Ratio } & \multicolumn{2}{c}{$\mathrm{CTE} \times 10^{-6}\left(\mathrm{~K}^{-1}\right)$} \\
\hline Mirror & Bar & $\mathrm{Si}$ & Brass & $\mathrm{Si}$ & Brass & $\mathrm{Si}$ & Brass \\
\hline 0.7 & 1.436 & 170 & 110 & 0.28 & 0.3 & 2.6 & 19 \\
\hline
\end{tabular}

TABLE II

Metal Mirror Assembly Simulation Parameters

\begin{tabular}{l|c|c|c|c|c|c|c}
\hline \multicolumn{2}{l|}{ Thickness $(\mathrm{mm})$} & \multicolumn{2}{c|}{ Young's Modulus $(\mathrm{GPa})$} & \multicolumn{2}{c|}{ Poisson's Ratio } & \multicolumn{2}{c}{ CTE $\times 10^{-6}\left(\mathrm{~K}^{-1}\right)$} \\
\hline Mirror & Bar & Invar & $\mathrm{Al}$ & Invar & $\mathrm{Al}$ & Invar & $\mathrm{Al}$ \\
\hline 1.1 & 1.0 & 148.4 & 69.0 & 0.3 & 0.35 & 4.0 & 24.0 \\
\hline
\end{tabular}

the grating waveguides and the material filling the gap, respectively. Substituting $\Delta L_{g} \rightarrow \Delta L_{g}+d \tan (\theta)$ in (2) a deviation of $\sim 0.13 \mathrm{GHz}$ from the design channel spacing of $100 \mathrm{GHz}$ was calculated for a total mirror tilt of $0.03^{\circ}$. This is consistent with the results of Fig. 3(a) where the wavelength separation between the two measured channels remained essentially constant over the entire range of angular rotation.

The condition for athermal operation of an R-AWG is obtained by differentiating (1), resulting in [8]

$$
\frac{\partial\left(n_{c}\right)}{\partial T} \Delta L+\left[\frac{\partial\left(n_{g}\right)}{\partial T} \Delta L_{g}(T)+\frac{\partial\left(\Delta L_{g}\right)}{\partial T} n_{g}(T)\right]=0 .
$$

In the vacuum, and approximately in air, the athermal operation condition (3) can be rewritten in terms of the angle $(\theta)$ between the external mirror and the reflecting surface of the R-AWG [8]

$$
\left(\frac{\partial \theta}{\partial T}\right)_{\text {ath }} \approx-\frac{1}{n_{g}} \frac{\partial\left(n_{c}\right)}{\partial T} \frac{\Delta L}{\frac{\partial\left(\Delta L_{g}\right)}{\partial \theta}}
$$

where $(\partial \theta / \partial T)_{\text {ath }}$ is the temperature-induced rotation rate of the external mirror making the device athermal and $\partial\left(\Delta L_{g}\right) / \partial \theta$ corresponds to the additional constant path length difference contribution added to the grating waveguides when angle $\theta$ is introduced between the external mirror and the AWG. Equation (4) shows that $(\partial \theta / \partial T)_{\text {ath }}$ does not depend on the initial angular position of the external mirror. The reflective AWGs used here have $\partial\left(\Delta L_{g}\right) / \partial \theta \sim 1.22 \mu \mathrm{m} / \mathrm{deg}$ With an air gap between the mirror and the device, we calculate using (4), that an angular temperature dependence of $-2.2 \times 10^{-4} \mathrm{deg} /{ }^{\circ} \mathrm{C}$ is needed in order to compensate for the change in the refractive index of the waveguides with temperature. If the gap medium between the AWG and the external mirror is filled with an index matching material, its refractive index temperature dependence must be taken into account in order to temperature compensate the device. Typical index matching materials have index-temperature dependence of the order of $-3.5 \times 10^{-4} /{ }^{\circ} \mathrm{C}$. Since temperature dependence of the index is negative, the rate of rotation of the external mirror should be reduced accordingly.

Equations (1)-(4) are valid for a constant separation $\left(d_{\mathrm{wg}}\right)$ between consecutive waveguides at the surface terminating the grating. All R-AWGs used in this work rely on this design. Non-constant values of $d_{\mathrm{wg}}$ would result in phase errors, producing additional loss and increased channel bandwidth penalties [10]. In addition, all the waveguides of the grating terminate perpendicularly to the reflecting surface.
A two-dimensional FE model was developed using ANSYS software package [11] to calculate the rate of rotation of the reflecting surface of composite mirrors with temperature. The purpose of this analysis is twofold: 1) to enable simulation of changes in the material and the geometry before prototypes are made, and 2) to examine methods for tuning the mirror assembly to provide fine adjustments in thermal response, without requiring major structural modifications. Finite-thickness quadrilateral plane-strain linear elastic elements were used to discretize the mirror body (ANSYS element type PLANE183). Tables I and II show the values of the material parameters used in the simulation of the composite mirrors of Fig. 1(b) and (c), respectively.

We showed in Fig. 5(b) that the mirror can be tuned by changing the position of the metal rod across the aperture in the Si body [see Fig. 1(b)]. A series of FE analyzes were performed to predict the dependence of the temperature response on this variable. An example of a FE simulation of a Si-mirror where a metal rod was positioned at $6.3 \mathrm{~mm}$ above the notch is shown in Fig. 7(a), for a $100^{\circ} \mathrm{C}$ temperature rise. The deformation is greatly exaggerated for easier visualization. As can be seen from the simulation, the reflecting surface of the mirror rotates with temperature. Furthermore, as is discussed in more detail below, the simulation predicts that the mirror surface remains planar. A comparison between simulated and measured temperature-induced mirror rotation rate as a function of the metal rod position $(h)$ above the notch is shown in Fig. 7(b). In this analysis, the gap between the external mirror and the device is assumed filled with air. The solid line shows the predicted $\partial \theta / \partial T$, while the open circles show the measured values. While a reasonable qualitative agreement is evident, the differences are larger than desired. This is attributed to the mechanical behavior of the adhesive, neglected in our model. This is also a major concern for the mirror design and fabrication. Properties of the adhesive are not well known, vary between types of adhesive, and are also potentially sensitive to temperature and humidity. This makes an accurate model of the mechanical behavior of this mirror assembly subject to many variables.

The performance of the model can be improved by more realistically representing the effects of the adhesive. We adjusted the effective elasticity of the metal bar to reflect the combined effect of the stiff bar and the softer epoxy, and used this parameter to improve the fit of the calculated $\partial \theta / \partial T$ over the full range of bar heights. The result is shown as a dashed line in Fig. 7(b). A qualitative agreement between the measured and calculated is obtained from either plot. The overall trend observed in the 


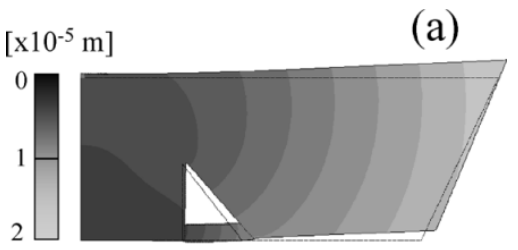

(b)

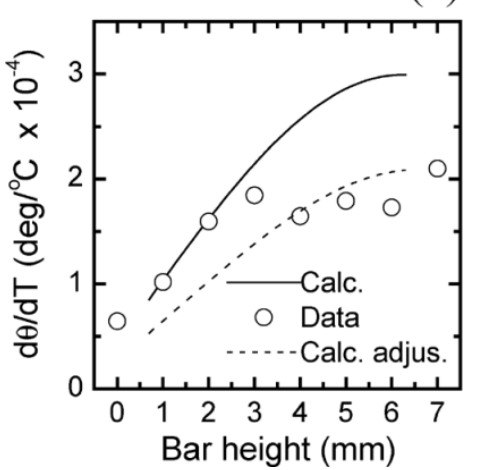

Fig. 7. (a) FE simulation for the Si composite mirror with a metal rod attached at $6.3 \mathrm{~mm}$ from the notch. (b) Experimental and simulated temperature-induced rotation rates for different positions of the metal rod across the aperture in the Si-body.

experimental and the calculated curves is a large increase of the rotation rate for $h<4 \mathrm{~mm}$. Larger values of $h$ resulted in increased value of $\partial \theta / \partial T$, but a lower rate.

An example of a FE analysis of the composite mirror with a solid Al disc, illustrated in Fig. 1(c), is shown in Fig. 8(a) for a temperature rise of $100^{\circ} \mathrm{C}$. The reflecting surface of the metallic mirror rotates with the temperature at a rate of $1.85 \times 10^{-4} \mathrm{deg} /{ }^{\circ} \mathrm{C}$, in agreement with the measured value of $1.26 \times 10^{-4} \mathrm{deg} /{ }^{\circ} \mathrm{C}$ obtained for the air-filled gap.

The composite metallic mirror can be also fine tuned, if required by variations in dimensions or material properties of the mirror, by replacing the solid disc shown in Fig. 1(c) with a ring. This additional fine tuning is illustrated in Fig. 8(b), which shows the thermally induced rotation rate calculated as a function of the ratio of the outer-to-inner radii of the ring. As the inner radius is increased the rotation rate increases, providing the desired tunability, without changing the mirror dimensions.

As mentioned above, for both mirror approaches (Figs. 7 and 8 ) the reflecting surface rotates without appreciable deformation. This is predicted in simulation, as shown in Fig. 9, where we plot the mirror deformation as a function of the surface coordinate at $100{ }^{\circ} \mathrm{C}$. The mirror is assumed to be perfectly flat at room temperature. From these curves, the maximum deviations from the flat surface are 0.26 and $0.01 \mu \mathrm{m}$ for the metal and the Si composite mirrors, respectively. The metal mirror deformation results in a predicted effective radius of curvature larger than $100 \mathrm{~m}$. This large radius of curvature would not produce any significant penalties in the device performance. The predicted Si mirror deformation is even lower. The reason for the difference is that in the Si composite design strain is concentrated in the region near the tip of the notch, while in the metal design the strain is distributed more evenly through the body of the mirror. Both approaches have some advantages. The concentrated strain maintains slightly better optical performance, but the distributed strain is less prone to failure. Deformation

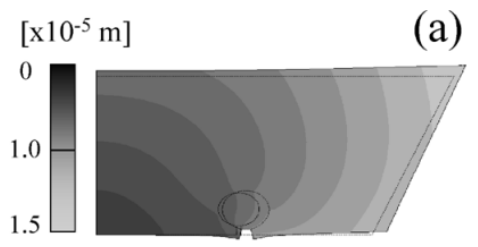

(b)

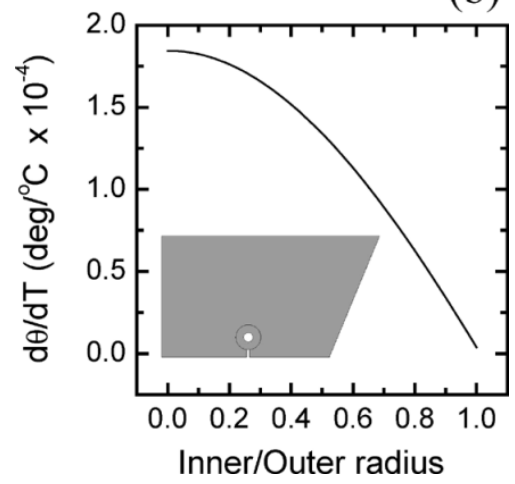

Fig. 8. (a) FE simulation for the metal composite mirror with an Al plug inserted into the aperture. (b) Simulated temperature-induced rotation rate for different values of the ratio inner/outer radii.

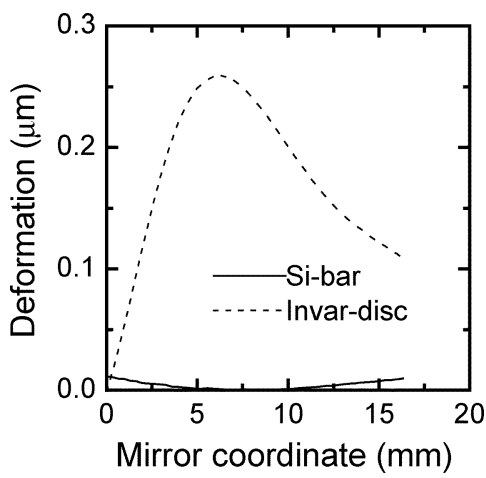

Fig. 9. Calculated deformation of the reflecting surface for both composite mirror approaches.

in the mirror surface will result in nonconstant changes in the optical path length of the waveguides, giving rise to phase errors that degrade the device performance [10]. As long as the average radius of curvature is kept above $\sim 80 \mathrm{~m}$, no such errors should be observed [12]. The simulation predictions are validated experimentally in Figs. 5 and 6 where the response of the R-AWG resulted in negligible device performance degradation over a wide range of temperature change, using both mirror approaches. Since the optical performance of both devices is acceptable, the metal design is preferred for lower peak strains and easier manufacture.

\section{CONCLUSION}

In summary, we show that combining an R-AWG with an external composite mirror results in accurate control of the channel wavelength and, at the same time, makes the device temperature insensitive. The center channel wavelength can be shifted by an amount equivalent to the channel-to-channel spacing without appreciable degradation of the device performance. Channel transmission peaks shift linearly with the angular position of the external mirror, in good agreement 
with simulations. The separation between consecutive output channels was found insensitive to the angular position of the external mirror.

The temperature sensitivity of silica-based R-AWGs was eliminated by designing composite mirrors fabricated with materials with different linear thermal expansion coefficients. Differences in the thermal expansion coefficients result in fixed-rate rotation of the reflecting surface of the mirror with temperature. We demonstrate athermal operation of R-AWGs using two different composite mirrors: 1) a silicon body with a metal rod attached across a machined aperture and 2) an invar body with a circular aperture filled with an $\mathrm{Al}$ plug. In the temperature range of $5-70^{\circ} \mathrm{C}$ both approaches reduced the temperature-induced wavelength-shift rate from $1.06 \times 10^{-2} \mathrm{~nm} /{ }^{\circ} \mathrm{C}$ to less than $3.0 \times 10^{-4} \mathrm{~nm} /{ }^{\circ} \mathrm{C}$. This was done without a significant degradation in the device performance.

A FE model was developed to investigate the influence of the material and geometric characteristics on the temperature-induced rotation rate of the reflecting surface. A discrepancy between measured and simulated rotation rate observed for the Si-mirror was attributed to the mechanical behavior of epoxy used in the attachment of the metal rod to the Si-body. A correction for this effect gave significantly improved results. Simulations predict that over a wide range of temperatures the reflecting surface of the mirrors described here do not deform enough to cause measurable phase errors. This is confirmed experimentally where no significant changes in the device performance were observed in the temperature range $5^{\circ} \mathrm{C}-70^{\circ} \mathrm{C}$. The numerical analysis demonstrates that fine tuning of the mirror rotation rate can be achieved by changing the position of the metal rod across the aperture machined in the Si-mirror, or by inserting metal rings with different inner radii in the invar mirror aperture.

\section{REFERENCES}

[1] A. Himeno, K. Kato, and T. Miya, "Silica-based planar lightwave circuits," IEEE Select. Topics Quantum Electron., vol. 4, pp. 913-924, Nov.-Dec. 1998.

[2] K. Okamoto, Fundamentals of Optical Waveguides. New York: Academic, 2000, pp. 264-269.

[3] C. Dragone, "Efficient Reflective Multiplexer Arrangement," U.S. Patent 5450511, Sept. 12, 1995.

[4] J. B. D. Soole, M. R. Amersfoort, H. P. LeBlanc, A. Rajhel, C. Caneau, C. Youtsey, and I. Adesida, "Compact polarization independent InP reflective arrayed waveguide grating filter," Electron. Lett., vol. 32, pp. 1769-1771, 1996.

[5] A. Kaneko, S. Kamei, Y. Inoue, H. Takahashi, and A. Sugita, "Athermal silica-based arrayed-waveguide grating (AWG) multi/demultiplexers with new low loss," Electron. Lett., vol. 36, pp. 318-319, 2000.

[6] N. Ooba, Y. Hibino, Y. Inoue, and A. Sugita, "Athermal silica-based arrayed-waveguide grating multiplexer using bimetal plate temperature compensator," Electron. Lett., vol. 36, pp. 1800-1801, 2000.

[7] J. Hasegawa, T. Saito, K. Tanaka, K. Nara, Y. Nekado, and K. Kashihara, "100 GHz-48ch athermal AWG with a novel temperature insensitive principle," in Proc. National Fiber Optics Engineers Conf., Orlando, FL, Sept. 2003, pp. 801-808.

[8] L. Grave de Peralta, A. A. Bernussi, V. Gorbounov, and H. Temkin, "Temperature insensitive reflective arrayed waveguide grating multiplexers," IEEE Photon. Technol. Lett., vol. 16, pp. 831-833, Mar. 2004.

[9] L. Grave de Peralta, A. A. Bernussi, S. Frisbie, R. Gale, and H. Temkin, "Reflective arrayed waveguide grating multiplexer," IEEE Photon. Technol. Lett., vol. 15, pp. 1398-1400, Oct. 2003.
[10] A. A. Bernussi, L. Grave de Peralta, and H. Temkin, "Electric field distribution in a grating of a folded arrayed-waveguide multiplexer," IEEE Photon. Technol. Lett., vol. 16, pp. 488-490, Feb. 2004.

[11] “ANSYS 8.0 Documentation,” ANSYS Inc., Canondale, PA, 2004.

[12] A. A. Bernussi, L. Grave de Peralta, V. Gorbounov, J. A. Linn, S. Frisbie, R. Gale, and H. Temkin, "Mirror quality and the performance of reflective arrayed waveguide grating multiplexers," J. Lightwave Technol., vol. 22, pp. 1828-1832, July 2004.

L. Grave de Peralta received the M.S. degree in physics from Oriente University, Cuba, in 1982 and the Ph.D. degree in electrical engineering from Texas Tech University, Lubbock, in 2000.

He was a Professor in the Department of Experimental and Theoretical Physics at Oriente University until 1989. Earlier work focused in vertical-cavity surface-emitting lasers design and material characterization (luminescence and $\mathrm{X}$-ray reflectivity). During the last three years, he has been working in planar light wave circuit design and development at Applied WDM Inc., Multipass Corporation, Ransom Canyon, TX, and Texas Tech University.

Ayrton A. Bernussi received the B.S., M.S., and Ph.D. degrees in physics from Universidade Estadual de Campinas (Unicamp), Campinas, Brazil, in 1981, 1984, and 1990, respectively.

In 1988, he joined the Optoelectronic Group at the Brazilian Telecommunication Company (Telebras), where he was involved in the development of semiconductor lasers and materials. From 1994 to 1995, he was a Postdoctoral Researcher at the Electrical Engineering Department, Colorado State University (CSU), Fort Collins. His research during this period was on high-temperature properties of strained quantum-well lasers. During 2000-2001, he was with the National Synchrotron Light Laboratory (LNLS), Campinas, Brazil, where he was involved in the development of semiconductor high-power lasers. Since 2001, he has been a Senior Research Associate at the Electrical Engineering Department, Texas Tech University, where he is involved in the development, characterization, and packaging of planar lightwave circuits.

V. Gorbounov received the B.S. degree in mechanical, electrical, and space engineering from Kiev International University of Civil Aviation, Kiev, Ukraine, in 1990. In 2003, he received the M.S. degree in electrical engineering from Texas Tech University, Lubbock, working in the design and fabrication of microopto-electro-mechanical systems with Prof. H. Temkin.

His current area of interest is the development of novel nanoscale devices for single molecular analysis such as DNA sequencing.

J. M. Berg received the B.S.E. and M.S.E. degree in mechanical and aerospace engineering from Princeton University, Princeton, NJ, in 1981 and 1984, respectively. He received the M.S. degree in mathematics and computer science and the Ph.D. degree in mechanical engineering and mechanics from Drexel University, Philadelphia, PA, in 1992.

He has held postdoctoral appointments at the U.S. Air Force Wright Laboratory, Dayton, $\mathrm{OH}$, and at the Institute for Mathematics and Its Applications, Minneapolis, MN. Since 1996, he has been at Texas Tech University, Lubbock, TX, where he is now an Associate Professor of mechanical engineering and a member of the Nano Tech Center. His research interests include control theory, and design, fabrication, and analysis of microsystems.

H. Temkin (SM'87-F'93) was a Postdoctoral Associate with Cornell University, Ithaca, NY, where he worked (with Prof. Fitchen) on optical properties of low-dimensional materials from 1975 to 1977.

In 1977, he joined the staff of AT\&T Bell Laboratories, Murray Hill, NJ, where he worked on a variety of optoelectronic devices, including light-emitting diodes (LEDs), buried heterostructure lasers, and quantum-well lasers, devices based on SiGe and high-speed bipolar transistors. He became a Distinguished Member of Technical Staff in 1985. In 1992, he joined the faculty of Colorado State University, Fort Collins, where he was Professor of Electrical and Computer Engineering. He moved to Texas Tech University, Lubbock, in 1996, where he serves as a Maddox Chair in Engineering. His research has focused on gas-source MBE growth of semiconductors with emphasis on ultraviolet photodetectors and LEDs based on $\mathrm{AlGaN}$ and passive devices for wavelength-division multiplexing. 